\title{
Lead Exposure Exacerbates Cardiovascular Risk
}

\author{
Praveen Sharma $\cdot$ Purvi Purohit
}

Published online: 13 April 2014

(C) Association of Clinical Biochemists of India 2014

Cardiovascular disease (CVD) is the leading cause of mortality all over the world. However, majority of CVDs are caused by risk factors that can be controlled, treated or modified, such as hypertension, cholesterol, overweight/ obesity, tobacco use, sedentary lifestyles and diabetes. Various environmental toxicants like Lead $(\mathrm{Pb})$ are also important preventable causes of variation in CVDs as reported in different human populations [1]. Although important measures have already been implemented in a number of countries to reduce environmental $\mathrm{Pb}$ exposure which includes the use of unleaded gasoline, $\mathrm{Pb}$ free paints, solder of canned foods, and glazed ceramics used for storage and preparation of food, it is still a major environmental health hazard in specific communities and targeted high-risk populations [2].

$\mathrm{Pb}$ intoxication has been found to be associated with hypertension and promotes atherosclerosis in experimental animals [2,3]. Hypertensive effects of $\mathrm{Pb}$ has also been documented in workers chronically exposed to high $\mathrm{Pb}$ levels [4]. Several epidemiological and clinical studies have found a link between chronic $\mathrm{Pb}$ exposure and elevated blood pressure [1]. Although a dose-response relationship between $\mathrm{Pb}$ exposure and blood pressure has also been reported however shape of the dose response relationship is not completely elucidated, especially at low levels of exposure. It is still not clear what should be the lowest level of $\mathrm{Pb}$ exposure that shall not be associated with $\mathrm{Pb}$ toxicity and its impact on blood pressure, although in the available studies there seems to be no evidence of a threshold effect [5].

P. Sharma $(\bowtie) \cdot$ P. Purohit

Department of Biochemistry, All India Institute of Medical

Sciences, Jodhpur, India

e-mail: praveensharma55@gmail.com
The precise mechanisms explaining a hypertensive effect of low chronic exposure to environmental $\mathrm{Pb}$ are unknown. Muntner et al. [6] has reported an inverse association between estimated glomerular filtration rate and blood $\mathrm{Pb}$ level at $\mathrm{Pb}$ concentration below $5 \mu \mathrm{g} / \mathrm{dl}$ in general population studies, indicating that $\mathrm{Pb}$-induced reductions in renal function could play a major role in hypertension. Enhanced oxidative stress, down-regulation of nitric oxide, stimulation of the renin-angiotensin system and soluble guanylate cyclase are among other potential mechanisms which could result in increased vascular tone and peripheral vascular resistance [5].

Metal-mediated formation of free radicals may enhance lipid peroxidation and changes in calcium and sulfhydryl homeostasis. $\mathrm{Pb}$ by promoting the production of reactive oxygen species, triggers a cycle of oxidative stress and inflammation in target tissues. Depletion of sulfhydryl reserves in cells provides an indirect mechanism for redox-inactive metals induced oxidative stress. These findings suggest a possible involvement of oxidative stress in the pathophysiology of $\mathrm{Pb}$ toxicity. Although oxidative stress is one of the important factor in the pathophysiology of $\mathrm{Pb}$ toxicity, it is not clear whether these alterations are the cause of the oxidative damage or a consequence of it. Increased expression and production of inflammatory markers in association with $\mathrm{Pb}$ exposure have also been reported in humans [2].

Most of the data available have been drawn from experimental studies and there are few cohort studies that evaluate the association of $\mathrm{Pb}$ with clinical cardiovascular outcomes in general population settings. The National Health and Nutrition Examination Surveys (NHANES) II and III showed a statistically significant increase in cardiovascular mortality with increasing blood $\mathrm{Pb}$ levels, although there was an overall decline in $\mathrm{Pb}$ levels in the US adults. Similarly the British Regional Heart study showed positive 
but non-significant association of coronary heart disease or stroke with elevated $\mathrm{Pb}$ levels. However, the unresolved issue is the impact of uncontrolled confounding and measurement error on the relative risk estimates in studies of $\mathrm{Pb}$ and clinical cardiovascular end points. Similarly the validity of occupational studies on $\mathrm{Pb}$ and cardiovascular mortality is limited by different methodological problems like bias of observations in occupationally exposed workers, which cannot be extrapolated to general population [5].

Various factors, both conventional and novel risk factors have been shown to be responsible for the morbidity and mortality of patients with cardiovascular diseases. No attention has so far been given to environmental toxicants and other trace elements such as $\mathrm{Pb}$, which can exacerbate the disease condition. A prospective pro-atherogenic effect, even if modest compared to other conventional risk factors would have a significant impact in sensitive population groups. In view of this it becomes important to explore the precise role and mechanism of $\mathrm{Pb}$ in development of atherosclerosis and CVD.

\section{References}

1. Navas-Acien A, Guallar E, Silbergeld EK, Rothenberg SJ. Pb exposure and cardiovascular disease-a systematic review. Environ Health Perspect. 2007;115:472-82.

2. Alissa EM, Ferns GA. Heavy metal poisoning and cardiovascular disease. J. Toxicol. 2011; doi:10.1155/2011/870125.

3. Silveira EA, Siman FD, de Oliveira Faria T, Vescovi MV, Furieri LB, Lizardo JH, Stefanon I, Padilha AS, Vassallo DV. Low-dose chronic lead exposure increases systolic arterial pressure and vascular reactivity of rat aortas. Free Radic Biol Med. 2014;67C:366-76. doi:10.1016/j.freeradbiomed.2013.11.021.

4. Skoczyńska A, Gruber K, Belowska-Bień K, Mlynek V. Risk of cardiovascular diseases in lead-exposed workers of crystal glassworks. Part I. Effect of lead on blood pressure and lipid metabolism. Med Pr. 2007;58(6):475-83.

5. Corradi M, Mutti A. Metal ions affecting the pulmonary and cardiovascular systems. Met Ions Life Sci. 2011;8:81-105.

6. Muntner P, Menke A, DeSalvo KB, Rabito FA, Batuman V. Continued decline in blood $\mathrm{Pb}$ levels among adults in the United States: the National Health and Nutrition Examination Surveys. Arch Intern Med. 2005;165(18):2155-61. 